\title{
Optimization of the MESH network route by means of an artificial neural model
}

https://doi.org/10.31713/MCIT.2020.29

\author{
Andrii Safonyk \\ National University of Water and Environmental Engineering \\ Institute of Automatics, Cybernetics and Computer \\ Engineering \\ Rivne, Ukraine \\ a.p.safonyk@nuwm.edu.ua
}

\author{
Maksym Mishchanchuk \\ National University of Water and Environmental Engineering \\ Institute of Automatics, Cybernetics and Computer \\ Engineering \\ Rivne, Ukraine \\ mishchanchuk_ak17@nuwm.edu.ua
}

\author{
Petro Lakus \\ National University of Water and Environmental Engineering \\ Institute of Automatics, Cybernetics and Computer Engineering \\ Rivne, Ukraine \\ lakus_ak15@nuwm.edu.ua
}

\begin{abstract}
This article about system of remote-control access to the rooms and buildings based on MESH network. On MESH network for make routes used neural network developed by Tensorflow and Keras.
\end{abstract}

Keywords-MESH network; neural network; networking; WiFi; IoT

\section{INTRODUCTION}

Today, we live in a world where most day-to-day tasks are simplified or automated and transferred to computer control. Remote control technologies are firmly entrenched in the life of modern man. These technologies not only help save time, but also allow you to not depend on location.

Internet of Things [1] (IoT) technology is often used to implement remote control, which involves the communication of various devices based on microcontrollers with each other and with remote servers. This concept allows such systems to work autonomously according to a pre-programmed algorithm without human intervention.

One of the tasks of remote management is access to the rooms (study area, performance of official duties, etc.), the solution of which will save a lot of time on obtaining permits and permits for access to the rooms.

The special relevance of the IoT concept is that there is a development of micro-electrical engineering, which requires exchange with its environment or with the information center. An example of IoT device connectivity is a Wi-Fi network with a MESH topology.

As you know on static routing no routing algorithm or update mechanisms are required. Therefore, extra resources (CPU and memory) are not required. [2]. But in turn, the more nodes in the network, the more difficult it is to build static routing in such a network. Therefore, the creation of a neural network that will do the appropriate routing is an urgent issue.

The following tasks were set for the implementation of a remote-control system based on the MESH network:

- to develop the system of remote access control to premises on the basis of MESH network;

- $\quad$ investigate the topology of the MESH network;

- develop and create a neural network to analyze the connections of MESH nodes and build the most efficient nodes.

\section{SYSTEM FOR REMOTE CONTROL OF ACCESS TO ROOMS}

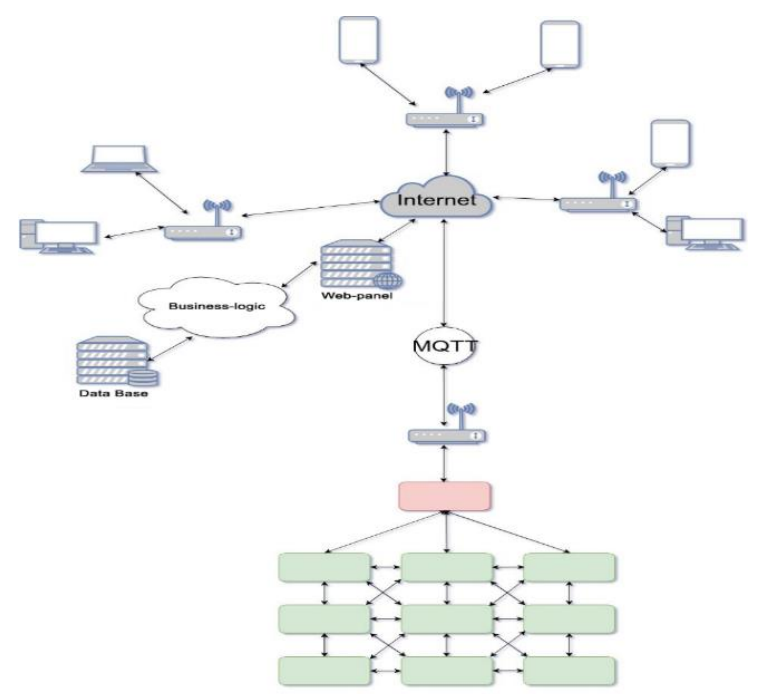

Figure 1. Scheme of the developed system 


\section{Modeling, control and information technologies -2020}

Suppose there are $n$ users who have access to $k$ room locks. Each user of the system is entered into a database with information about which room he has access to. The database is located locally on each node, all changes are made through the WEB-panel, the database is updated using the MQTT protocol. In a situation where there is no Internet access, the database is available for verification, but there is no possibility to update the data. When the user identifies himself with his private key, the key number is checked with the database and in case the user has access to the room, the door will be open for him, if not it will remain closed.

The advantage of such a system is that the database update is not performed by changing the software version of each local station database, but by updating data from the server via the Internet. Also, this system provides a separate autonomous power supply if power is not available in the building, the system will continue to operate.

The implemented system is divided into two logical modules: server and client part.

The server part is implemented using ReactJS to write the WEB-interface and programming language PHP 7 as a database relational database MySQL.

Using ReactJS [3], a SPA application was created. SPA is a web application that fits on one page to provide the user with an experience close to using a desktop application. Since this architecture allows the user to feel as if he is using a desktop application, which is common to most users, so to create a control panel, this architecture was used.

To interact with the client control panel, namely SPA used REST API backend part which was written in PHP programming language using the following structure: a controller that receives a request from the client and transmits validated data to the model, which processes the received data and stores them in the database MySQL data. To do this, an ORM system such as Active Query was used. ORM system is a system that allows you to present data from a database as an object for further processing by business logic.

IoT technology and MQTT protocol were used to interact business logic and the client part (the part located indoors). The principle of this protocol is the principle of subscription, in the client subscribes to updates in a particular topic and when another client publishes a message in this topic, this message will be sent to all clients who subscribe to this topic and further process the data. Therefore, the client part has devices based on microcontrollers ESP8266 or ESP32 connected to the Internet and signed for updates in the topic in which the server part publishes configuration changes for each individual station. Next, the station receives the database update data, processes it and stores it in its local database.

MESH network communication topologies were used to connect each station to the Internet. The scheme of the developed system is shown in figure 1 .

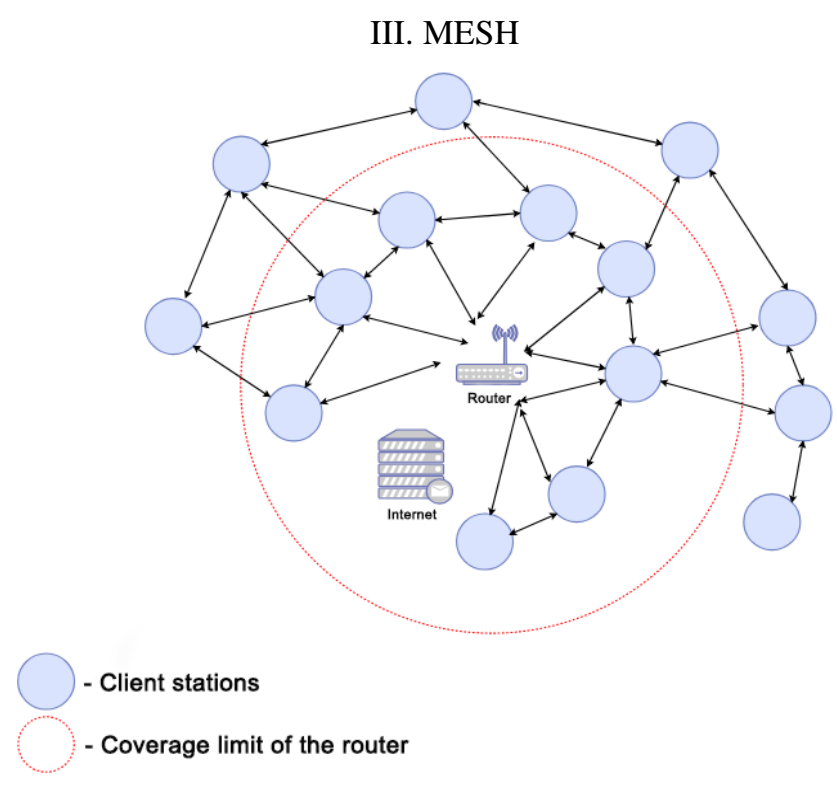

Figure 2. MESH topology

MESH is a network topology where each node is connected to each other and acts as a switch for other nodes [4]. This topology is difficult to implement but provides high fault tolerance in its work in the event that one of the points fails, this network can continue to operate, because it will be reformed and will continue to transmit data in the middle.

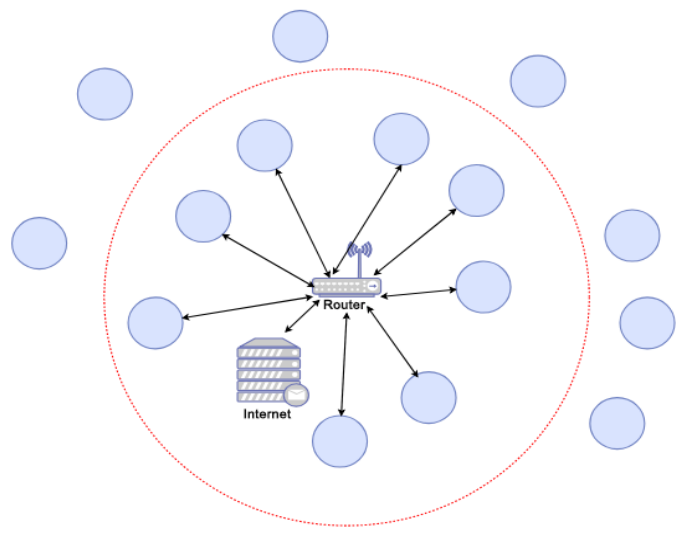

- Client stations

- Coverage limit of the router

Fig. 3. Traditional network architecture 
Modeling, control and information technologies -2020

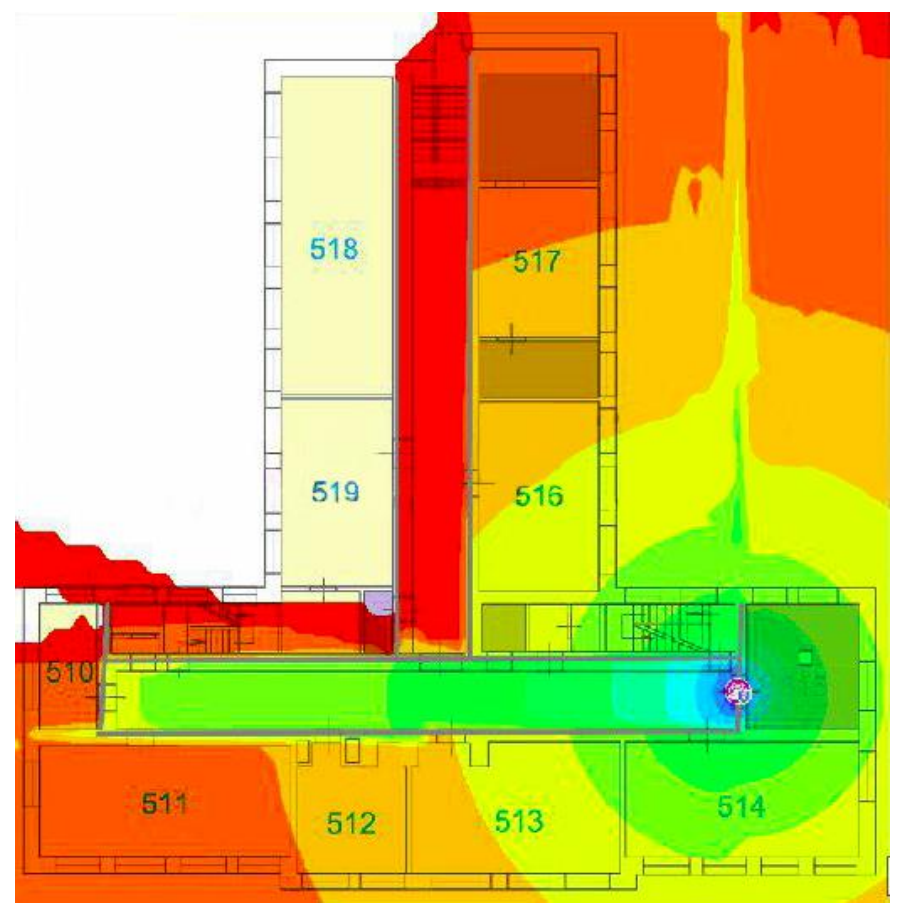

Figure 4. Signal propagation in the standard wireless network architecture.

Also, this network does not have such a disadvantage as a standard topology, when there is one main router signal coverage area which is limited by the maximum allowable power standards of its transmitter, its technical features and obstacles such as: different walls that create obstacles to signal transmission (see Figure 3).

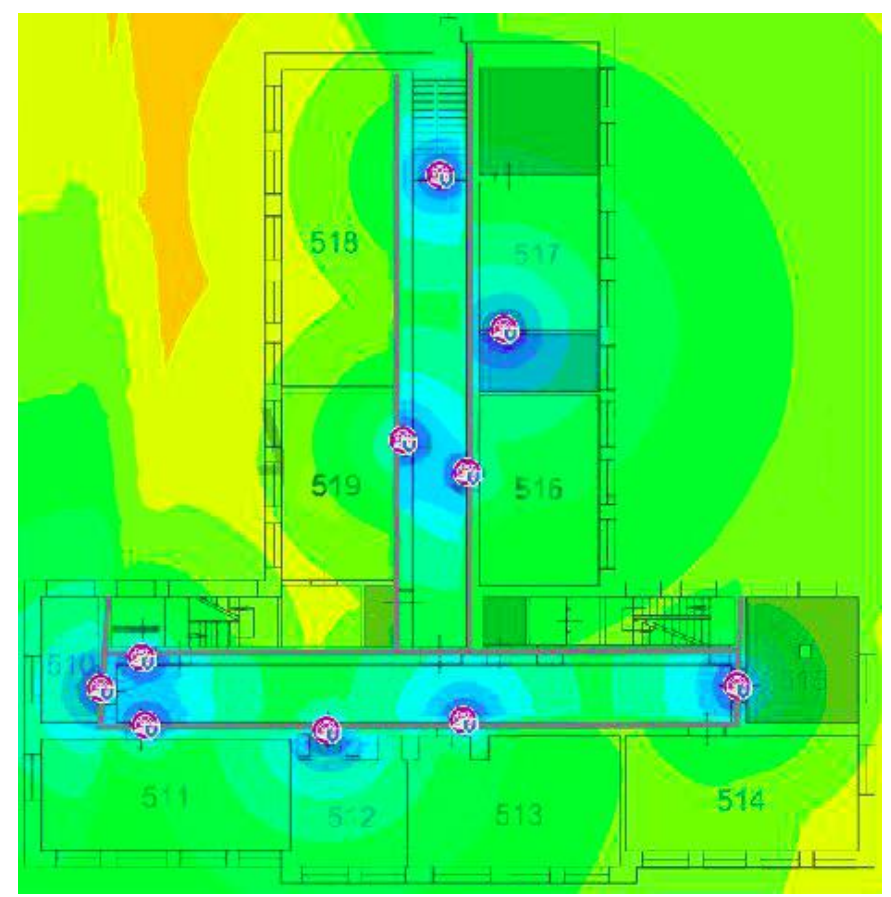

Figure 5. signal propagation in the MESH network.
In the MESH topology, each point is a switch and amplifies the signal of each other (see Figure 4) so you can avoid the problem when the signal strength of the main station

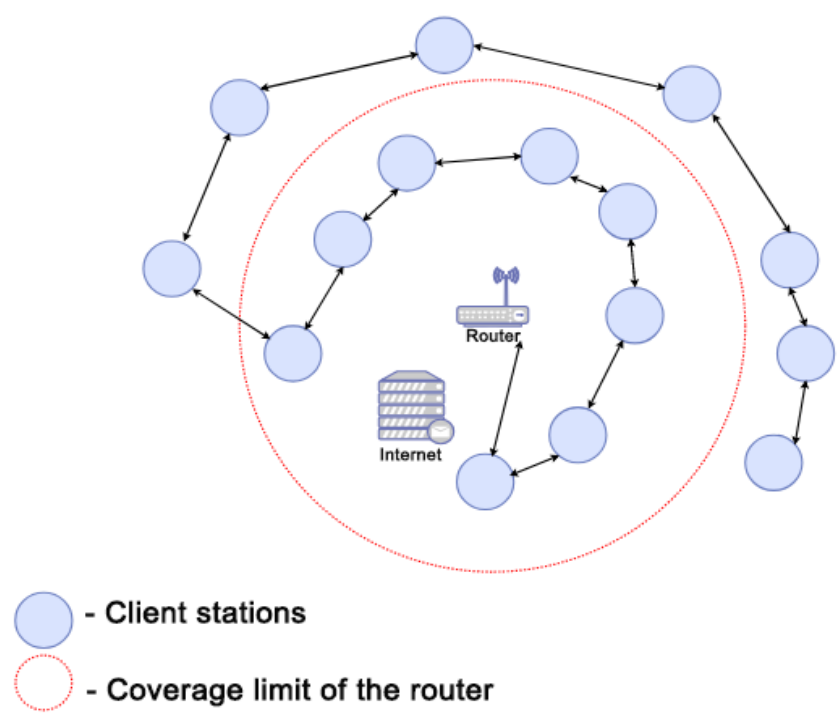

Fig. 6. Wireless repeater architecture

In comparison of MESH topology with topology of wireless repeater MESH there is no clear one static route for transfer from one point to the next as they are not connected in series (see fig. 5 ), and joins points with the most optimum parameters.

To solve this problem, it was decided to develop a neural network that would build routes in the MESH network.

\section{MESH AND NEURAL NETWORK}

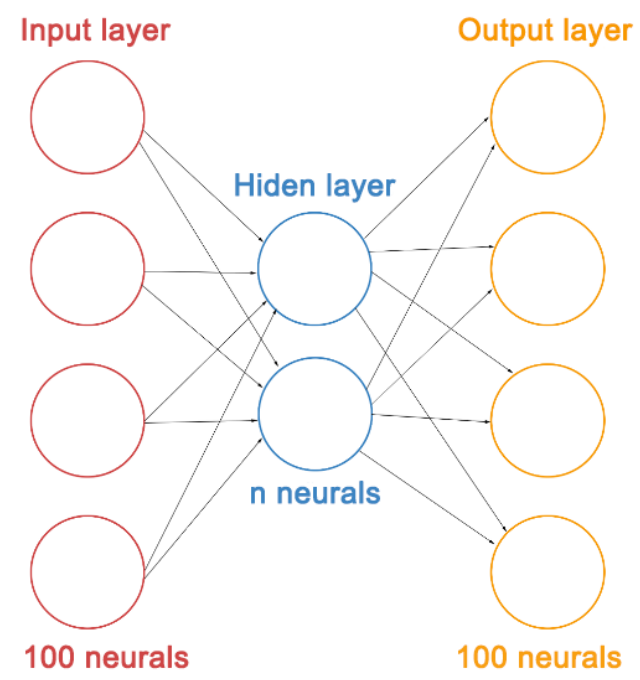

Fig. 7. The structure of the neural network

MESH networks have a major drawback is the complexity of their construction to solve this problem a neural network has been developed to build static routes. The neural network has 3 layers (see Figure 6): input, output and hidden. Each neuron is 


\section{Modeling, control and information technologies -2020}

connected to each neuron in the next layer. The first input layer receives the vector of network connections with the signal strength parameter to the next station. Then the data enters the hidden layer where it is processed and transferred to the source layer. The result is a vector with the probability of selecting a particular connection to transfer data between nodes.

The following Keras, Tensorflow [5], NetworkX components and Python programming language version 3.6 are used to implement the neural network.

Keras - a high-level neural network API written in Python and capable of working on TensorFlow, CNTK or Theano.

TensorFlow is an open source platform for machine learning. With a comprehensive, flexible ecosystem of tools, libraries, and resources that makes it easy to create and deploy machinelearning-driven applications.

NetworkX is a Python package for creating, manipulating and studying the structure, dynamics and functions of complex networks.

NetworkX provides:

- $\quad$ tools for studying the structure and dynamics of social, biological and infrastructural networks;

- $\quad$ standard programming interface and graphics implementation, which is suitable for many programs.

To determine the number of neurons in the hidden layer, an experiment was performed in which the mistakes was determined depending on the number of neurons as can be seen from table 1, that for a neural network 500 neurons are enough.

Table 1 . The results of the study of the optimal number of neurons

\begin{tabular}{|c|c|c|c|c|c|}
\hline $\begin{array}{c}\text { Number } \\
\text { of } \\
\text { neurons }\end{array}$ & 100 & 500 & 1000 & 1500 & 2000 \\
\hline Mistake & 0.0182 & 0.0152 & 0.0154 & 0.0152 & 0.0168 \\
\hline
\end{tabular}

To create a neural network, it was necessary to determine the optimal function of activation of the hidden layer. For this purpose, the 8 most used functions were selected and their work was compared.

A study was conducted in which each function was used as an activation function. A study base of 2,500 examples and a test base of 500 examples were generated for the study.
After training and testing of the neural network, the following results were obtained for neural networks with all the studied functions, there is still the possibility of learning. The mistake for the functions is shown in table 2.

Table 2. The results of determining the optimal activation function

\begin{tabular}{|c|c|}
\hline Function & Mistake \\
\hline elu & 0.0367 \\
\hline Exponential & 0.0147 \\
\hline Linear & 0.0379 \\
\hline Sigmoid & 0.0164 \\
\hline relu & 0.0353 \\
\hline selu & 0.0281 \\
\hline softplus & 0.0501 \\
\hline softsign & 0.0382 \\
\hline
\end{tabular}

As can be seen from the results for this task, the exponential function is best suited and therefore used in the neural network to build routes in the MESH network.

Therefore, based on the research, it was shown that the MESH network has significant advantages for the implementation of the remote access task among other topologies of wireless networks. To improve routing, a neural network was created and the optimal activation function was selected.

\section{REFERENCES}

[1] Samuel Greengard, The Internet of Things, Cambridge, MA: MIT Press, 2015, $232 \mathrm{pp}$.

[2] Networking Academy's Introduction To Routing Dynamically. Cisco Academy, 2014. [online] Ciscopress.com. Available at: http://www.ciscopress.com/articles/article.asp?p=2180210\&seqNum=12

[3] R. Wieruch, The Road to React. 2018, $246 \mathrm{p}$.

[4] K. Kumaravel, V. Renugadevi, A. Marimuthu. A study on protocols in wireless mesh network, International Journal of Computer Science \& Engineering Technology, Vol. 6 No. 07, 2015, P. 413-415.

[5] Geron Aurelien. Hands-on Machine Learning with Scikit-Learn, Keras, and TensorFlow: Concepts, Tools, and Techniques to Build Intelligent Systems. 2nd Edition. O'Reilly, 2019, 856 p. 